\title{
Right inferior phrenic artery; Don't miss on MDCT before chemoembolization of hepatic dome HCC
}

\author{
Mohamed Saied Abdelgawad ${ }^{*}$ and Rasha Abdelhafiz Aly
}

\begin{abstract}
Background: Transarterial chemoembolization (TACE) is considered a standard local-regional treatment for intermediate-stage hepatocellular carcinoma (HCC) and the most common bridging therapy. HCC typically receives its blood supply from the hepatic artery; however, it can recruit a parasitic supply from extrahepatic collateral arteries. The right inferior phrenic artery (RIPA) is considered the most common extrahepatic collateral pathway supplying HCC and so interfere with the therapeutic efficacy of TACE resulting in treatment failure and poor outcome. Imaging by MDCT with angiography has an essential role in depicting the presence or absence of an EHC arterial supply during the pre-TACE evaluation. We analyzed MDCT studies with CT angiography of patients with hepatic dome HCC retrospectively to confirm the diagnosis of HCC and to assess its blood supply. All patients were subjected to a proper assessment by history, clinical examination, as well as routine laboratory investigations

Results: Our study includes 58 cases with hepatic dome HCC. On CT angiography, the extrahepatic collateral from the RIPA supplying hepatic dome HCC was detected in about 33 out of 58 cases (56.9\%). The RIPA arose directly from the aorta in 30 cases (90.9\%) with only three cases (9.1\%) from the celiac trunk. All cases were managed with TACE with about 25 out of 33 cases (75.8\%) with extrahepatic RIPA were undergone concomitant embolisation of both RIPA and hepatic artery during TACE procedure.
\end{abstract}

Conclusion: MDCT assesses well the HCC arterial supply which is fundamental for planning subsequent cannulation and embolization of extrahepatic RIPA supply to hepatic dome HCC.

Keywords: Hepatic dome, HCC, MDCT, RIPA, Chemoembolization

\section{Background}

In general, the anatomical site of hepatic dome refers to the liver parenchyma adjacent to the diaphragm. The hepatic dome is related on the anterior, lateral, and posterior aspects to diaphragm, lung parenchyma with the covering pleura, and thoracic cage (Fig. 1). On the medial aspect, the hepatic dome is related to the gastric cardia and inferior vena cava (IVC) anteriorly and the vertebral column posteriorly [1].

Definite lesion localization within the hepatic dome is critical, especially during management to optimize the

* Correspondence: mselgawad@yahoo.com

Radiology Department, National Liver Institute, Menoufia University, Shebin Elkoum, Egypt

\section{Springer Open}

diagnostic yield and provide perfect tumor destruction. Accurate characterization of lesions on multidetector computed tomography (MDCT) can be attained by either use of anatomic landmarks, contrast administration, or additional methods as maximum intensity projection (MIP) and multiplanar reformation (MPR) [1].

Hepatocellular carcinoma (HCC) is one of the most common widespread malignancies and considered the third cause of cancer deaths [2]. HCC usually develops on a background of liver cirrhosis. However, non-cirrhotic liver and sometimes a healthy liver may be involved [3].

Transarterial chemoembolization (TACE) is considered a standard local-regional management for most patients with unresectable HCC [4] and has become a valid 


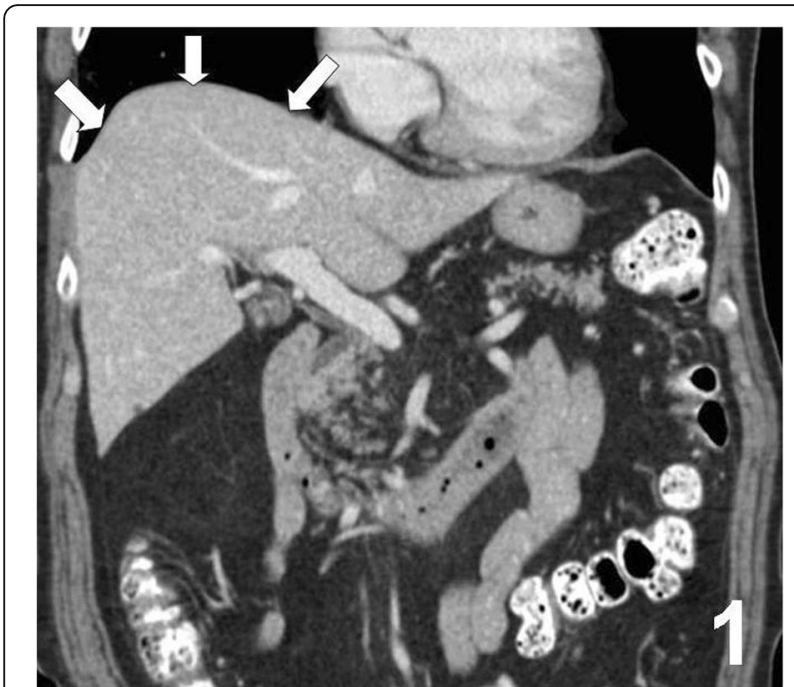

Fig. 1 Anatomy of the hepatic dome. Coronal reformatted computed tomography image demonstrating the anatomical site of the hepatic dome (arrows)

alternative to hepatic surgery [5]. Familiarity with variations in the hepatic arterial anatomy is essential to accomplish embolization. In some patients, in addition to anatomic variations of the hepatic arteries, various extrahepatic collateral $(\mathrm{EHC})$ arteries that may provide a partial or total vascular supply to HCC in certain conditions can obtain TACE technically challenging [6].

The right inferior phrenic artery (RIPA) is considered the most prevalent extrahepatic collateral pathway supplying peripherally located HCCs, even when the hepatic artery is patent $[7,8]$. RIPA generally arises from the aorta or celiac axis independently or as a common trunk with the left inferior phrenic artery. Knowing the anatomy and variations of the RIPA is crucial prior to the interventional radiological procedures concerning RIPA [9].

Embolization of the RIPA has been reported as adjunctive therapy to hepatic artery embolization and this can be done without critical technical complications. A few minor complications may occur, such as shoulder pain, minimal pleural effusion, basal atelectasis, and transient mild hemoptysis; because the RIPA is one of the major arterial supply to the diaphragm [10].

The variable anatomy of the RIPA origin can be challenging for its manipulation and in this context preprocedural MDCT with angiographic mapping of RIPA anatomy can be helpful in planning conventional angiography and subsequent embolization [10].

Therefore, the interventional radiologist prior to TACE should be familiar with the variable EHC arterial supplies to HCC and how to properly interact with these cases [6]. Consequently, we can save angiographic time, curtail the use of contrast media, and limit patient exposure to radiation by good MDCT arterial mapping [7].

\section{Patients and methods}

Patients characteristics

An institutional review board approved this study and decided that informed consent was not required. Between July 2017 and December 2019, we retrospectively evaluated patients referred for triphasic liver computed tomography $(\mathrm{CT})$ to assess hepatic focal lesion.

We select patients with hepatic dome HCC. These patients were subjected to routine history taking, physical examination, liver function and serum $\alpha$-fetoprotein (AFP) tests, ultrasonography, and abdominal enhanced CT imaging. The diagnosis of HCC was made by a combination of imaging $\mathrm{CT}$ findings and evaluation of the specific tumor marker (AFP).

The treatment strategies of HCC were selected through discussion by hepatologists, surgeons, and diagnostic and interventional radiologists. Therapeutic chemoembolization (TACE) was selected for inoperable HCC and based on performance status, hepatic functional reserve, the size or number of lesions and patients' wishes.

\section{CT technique}

The examination was performed using 128 MDCT scanner; (Toshiba Medial system, Aquilion, Japan). Then, 20 gauge cannula was used for venous accesses at the upper limb though the antecubital vein preferably on the right side. Slice thickness of $0.5 \mathrm{~mm}$ at 0.25 interval was used for reconstruction; with $120 \mathrm{kV}$ and $225 \mathrm{~mA}$. A revolution time of $0.4 \mathrm{~s}$ was used. Dynamic contrast injection of $120-150 \mathrm{ml}$ nonionic iodinated contrast material was used at a rate of 4-5 $\mathrm{ml} / \mathrm{s}$ though a mechanical injector "Stellant, MEDRAD."

Bolus tracking of contrast was used after delay of $6 \mathrm{~s}$ after reaching the preset aortic attenuation value at about $150 \mathrm{HU}$ to start the arterial phase imaging. Then

Table 1 The characteristics of hepatic dome HCCs at the initial MDCT examination in our studied cases

\begin{tabular}{ll}
\hline Hepatic dome HCC & No. of cases (58) \\
\hline Tumor size & $12(20.7$ \\
$\cdot<5 \mathrm{~cm}$ & $39(67.2)$ \\
$\cdot 5-10 \mathrm{~cm}$ & $7(12.1)$ \\
$\cdot \geq 10 \mathrm{~cm}$ & \\
Tumor multiplicity & $50(86.2)$ \\
$\cdot$ Single & $8(13.8)$ \\
- Multiple & \\
Visualized pseudocapsule & $22(37.9)$ \\
$\cdot$ With pseudocapsule & $36(62.1)$ \\
- Without pseudocapsule & \\
Tumor growth pattern & $35(60.3)$ \\
- Non-exophytic & $23(39.7)$ \\
• Exophytic &
\end{tabular}

Percentages/total in parentheses 


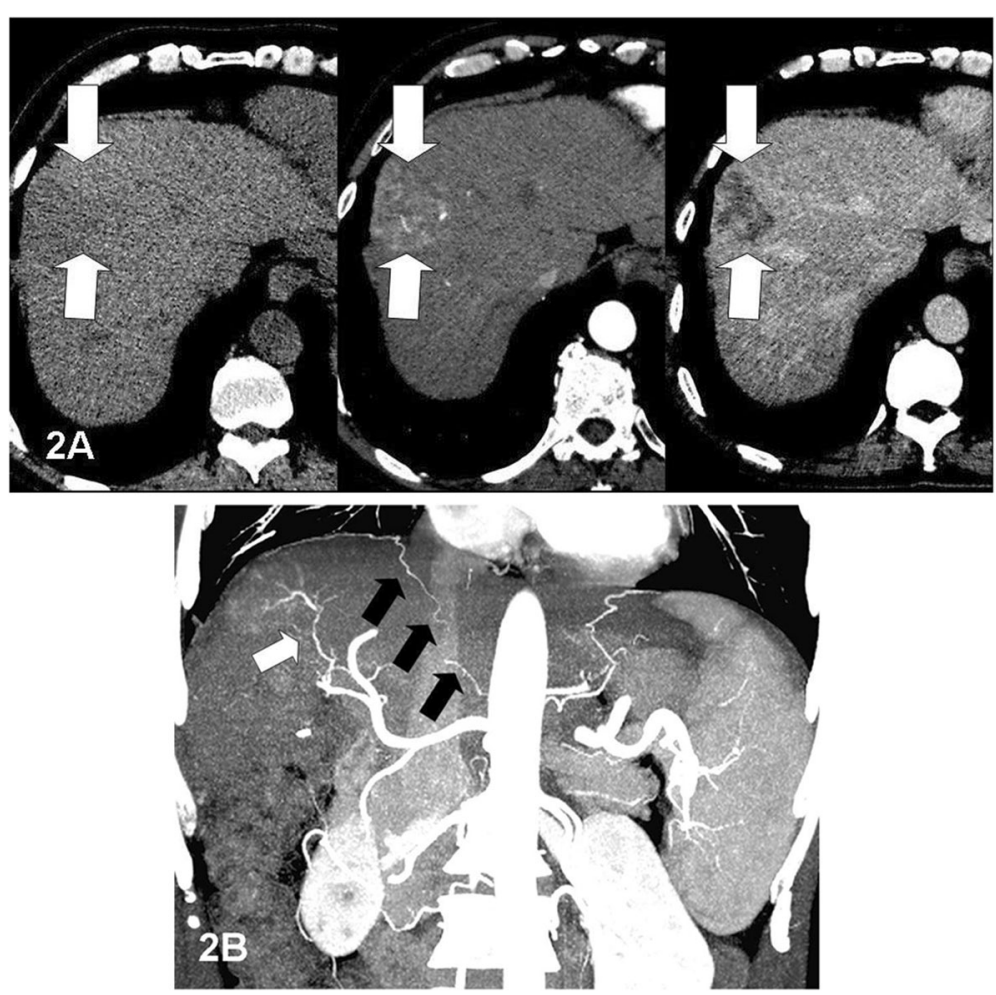

Fig. 2 Initial multi-phase CT liver with a axial non-contrast, arterial and delayed phases showing HCC (white arrows) at segment VIII with wash-in wash-out contrast dynamics. b Coronal MIP showing double arterial supply of the lesion by the right hepatic artery (white arrow) and by extrahepatic right inferior phrenic artery (black arrows)

portal and venous phases were scanned at about $70 \mathrm{~s}$ and 180-s delay time respectively, calculated from the start time of contrast material injection.

MDCT angiography was performed by post processing of the raw data and projected though three-dimensional option, multi-planar reconstruction and maximum intensity projections (MIP) of the arterial phase images. The origin of the right phrenic artery from the aorta or celiac trunk was recorded.

\section{Data analysis}

All multidetector CT data were transferred from the archive to a workstation (Vitrea 2.2 or Osrix), via internal network connections. All images were reviewed by two radiologists to assess all patients with hepatic dome HCC. The selected patients were examined prior to and/or after TACE to confirm the tumor size, activity, and its vascular supply.

The diagnosis of HCC should be made on a dynamic multiphasic contrast-enhanced CT study. The presence of arterial enhancement with subsequent wash-out on portal or delayed phases are considered to be the definitive imaging features of HCC. Delayed enhancing capsule can also be identified as a feature of HCC.

On arterial phase CT scans and CT angiography, we can frequently identify the vascularization of $\mathrm{HCC}$. Although most HCCs are supplied by the hepatic artery or its branches, they may receive blood supply from extrahepatic arteries (EHAs) which are initially "non-hepatic" in destination.

There are two reasons for this: the tumor location within the liver and previous management which lead to

Table 2 Extrahepatic right inferior phrenic artery supplying hepatic dome HCC on CT angiography

\begin{tabular}{llllll}
\hline Extrahepatic & \multicolumn{2}{l}{ Time of occurrence } & & & Site of origin \\
\cline { 2 - 3 } RIPA (33/58) & On initial CTA & On Follow-up CTA & & From abdominal aorta & From celiac axis \\
\hline No. of cases & 18 & 15 & 30 & 3 \\
\% to the total 33 cases with RIPA. & 54.5 & 45.5 & 25.9 & 50.9 & 9.1 \\
\% to the total 58 hepatic dome HCC cases & 31 & 51.7 & 5.2 \\
\hline
\end{tabular}


Table 3 Prevalence of Extrahepatic RIPA feeder according to tumor characteristics and chemoembolization session

\begin{tabular}{lll}
\hline Parameters & No and \% in relation to the 33 cases with RIPA & \% in relation to the total 58 HCC cases \\
\hline Tumor size & $0(0)$ & 0 \\
$\cdot<5 \mathrm{~cm}$ & $29(87.9)$ & 50 \\
$\cdot 5-10 \mathrm{~cm}$ & $4(12.1)$ & 6.9 \\
$\cdot \geq 10 \mathrm{~cm}$ & & 32.8 \\
$\cdot$ Exophytic & $19(57.6)$ & 24.1 \\
$\cdot$ Non-exophytic & $14(42.4)$ & Tumor growth pattern \\
Chemoembolization session & & 18.9 \\
$\cdot$ Single & $11(33.3)$ & 37.9 \\
- Repeated & $22(66.7)$ &
\end{tabular}

Percentages/RIPA cases in parentheses

changes in tumor vascularization as hepatic arterial flow interruption by repeated chemoembolization.

As the inferior phrenic artery supplies most of the diaphragm, including the area in contact with the bare area of the liver. Subsequently, the right inferior phrenic artery was found to be the most common extrahepatic arterial feeder of the hepatic dome HCC. With CT angiography, we can easily identify the segment of the parasitic right inferior phrenic artery to the hepatic lesion and suggest its origin.

In the present study, the patients undergone TACE procedure through embolization of the hepatic artery only, where the lipiodol retention did not cover the entire tumor or there was a focal defect in the tumor within the lipiodol deposition; other potential tumorsupplying arteries were sought. This can be confirmed by the selective conventional angiography of the suspected extrahepatic ectopic artery during the procedure.

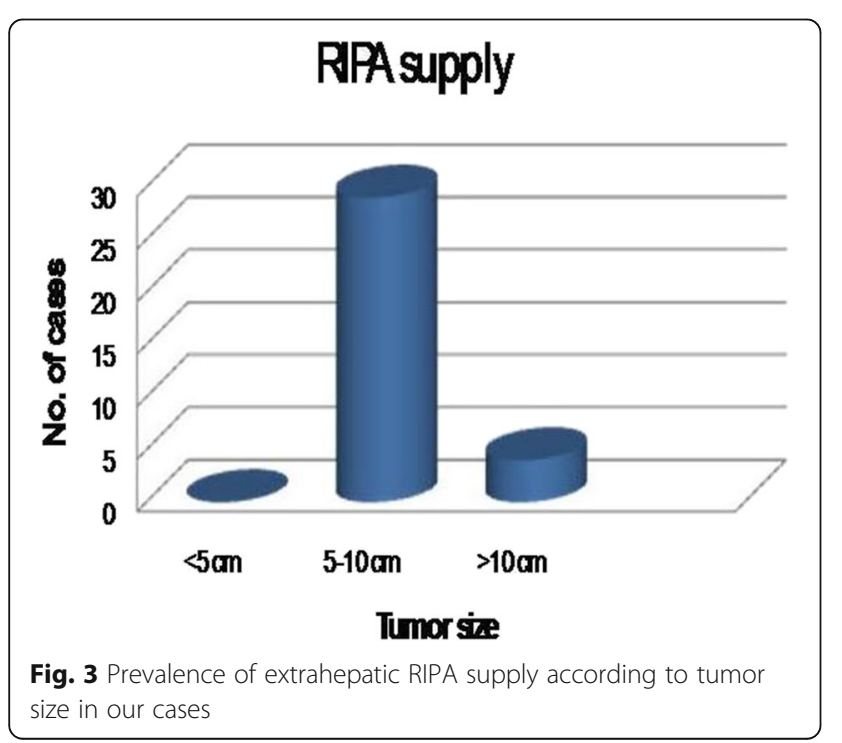

\section{Statistical analysis}

The data collected were tabulated and analyzed by the Statistical Package for Social Science (SPSS) version 25.0. Parametric data were presented with mean \pm standard deviation and non-parametric data with median and range. Categorical data were presented with frequency and percentage. Screening of patients with hepatic dome HCC with identified extrahepatic arterial supply will be done with Chi-square or Fisher's exact test as appropriate.

\section{Results}

In this cohort study, 58 patients with hepatic dome HCC were analyzed for extrahepatic collateral arterial supply by the right inferior phrenic artery (RIPA). It includes 40 males and 18 females with their mean age of 58.45 years and ranging between 45 and 71 years old.

Within our cases, three out of 58 (5.2\%) were classified as Child-Pugh class A, $36(62 \%)$ as class B, and 19 (32.8\%) as class $\mathrm{C}$ at the time of tumor embolization. The etiology of liver disease was found to be due to hepatitis C virus in 47 patients (81\%) and hepatitis B virus in eight patients $(13.8 \%)$, while the etiology was not proven in three patients (5.2\%).

Our cases were presented with hepatic dome HCC at the initial triphasic liver CT examination. All lesions are grouped according to the tumor size, multiplicity, visualized pseudocapsule, and tumor growth pattern as shown in Table 1. According to the status of pseudocapsule, lesions were divided into two groups: groups with (22/58) and without intact pseudocapsule (36/58). The criterion for intact pseudocapsule was complete delayed enhancing ring around the lesion which was isodense in the non-contrast scan.

According to the history of tumor therapy, all patients were managed with TACE with only seven out of $58 \mathrm{pa}-$ tients (12.1\%) had a history of previous percutaneous radiofrequency ablation (RFA) of a hepatic dome HCC. Within our study, 26 out of 58 patients (44.8\%) showed a history of repeated chemoembolization sessions. 

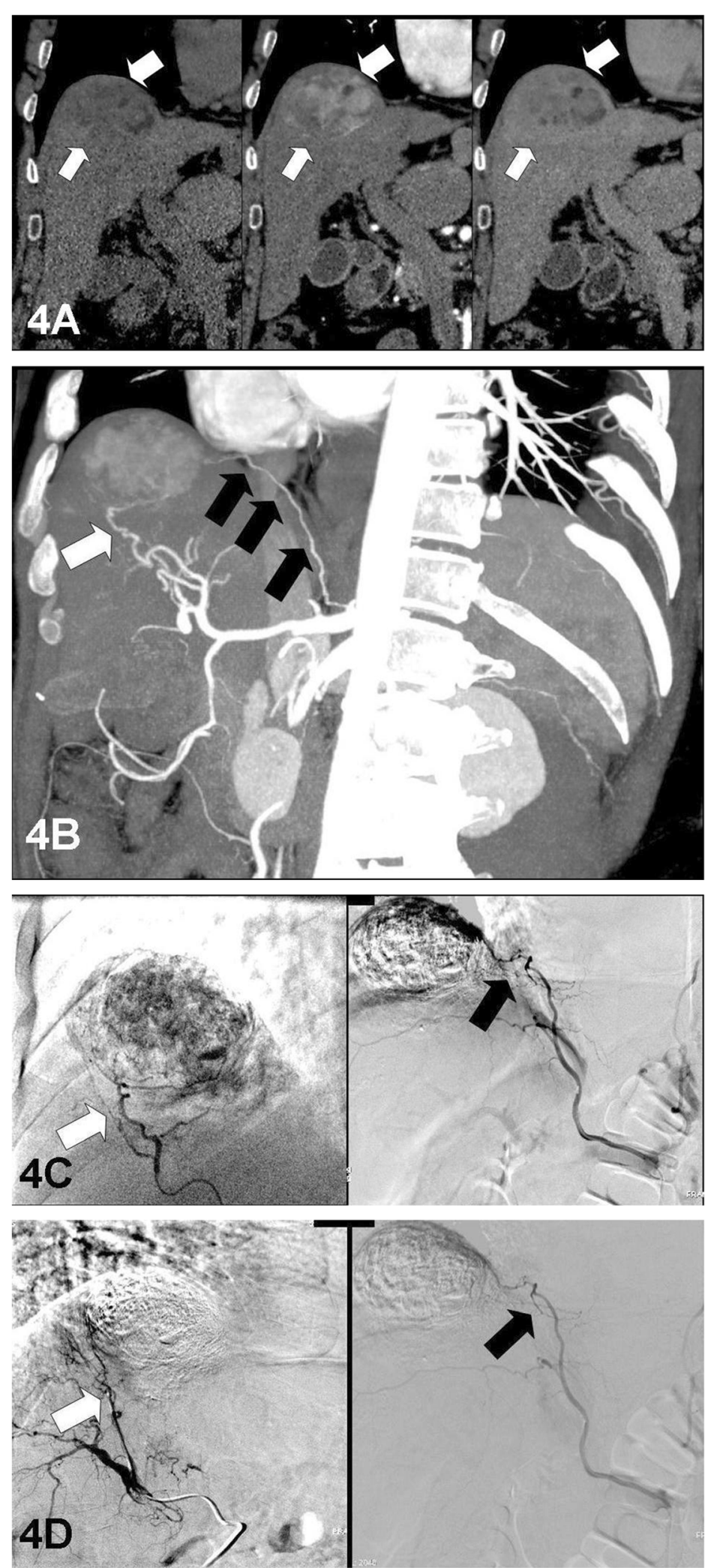

Fig. 4 (See legend on next page.) 
(See figure on previous page.)

Fig. 4 Initial multi-phase CT liver with a coronal non-contrast, arterial and delayed phases showing a well-defined hepatic dome HCC (white arrows) with heterogeneous arterial enchantment and delayed wash-out. b Coronal oblique MIP showing arterial supply of the lesion by both the right hepatic artery (white arrow) and by extra-hepatic right inferior phrenic artery (black arrows). c Selective conventional angiogram of the right hepatic (white arrow)and right superior phrenic arterial (black arrow) supply to the hepatic dome HCC showing tumor hypervascularity, followed by $\mathbf{d}$ embolization of both arteries (white and black arrows) with consequent no tumor staining could be seen

Of total cases, extrahepatic arterial supply through right inferior phrenic artery (Fig. 2) was discovered on CT angiography in 33 out of 58 patients (56.9\%). Within our 33 patients with extrahepatic RIPA supply, 18 patients (54.5\%) were found on initial CT angiography (CTA), while 15 out of 33 (45.5\%) were discovered with follow-up CT (Table 2).

On CT angiography, the RIPA arose directly from the aorta in 30 out of 33 cases (90.9\%) with only three out of 33 (9.1\%) from the celiac trunk. The prevalence of ectopic blood supply in our patients with different tumor sizes and tumor growth pattern as well as number of chemoembolization sessions are summarized in Table 3. In general, the larger the tumor, the more liability of the lesion to be fed by ectopic arteries (Fig. 3) as well as tumor with exophytic pattern.

In this study, 25 out of 33 cases (75.8\%) with extrahepatic RIPA supplying hepatic dome HCC were undergone concomitant embolization of RIPA (Figs. 4 and 5) during TACE procedure. Post-procedure triphasic CT revealed lipiodol deposition along part of the tumor supplied by the RIPA. While the remaining eight cases (24.2\%) had only hepatic artery embolization with non-embolized ectopic RIPA (Fig. 6) during TACE procedure.

The previously described eight cases with nonembolized extrahepatic RIPA underwent follow-up triphasic CT liver examination with consequent progressive course of the active tumor component (Figs. 7 and 8) supplied by the parasitic RIPA, associated with continued elevation of serum $\alpha$-fetoprotein levels.

During or immediately after the procedures treated via the right IPA, all 25 patients complained of right shoulder pain with about 23/25 patients (92\%) complained of hiccups with gradual improvement within a few days by conservative treatment. Minimal right-sided pleural effusion was observed on follow-up CT 1 month later in about $17 / 25$ cases (68\%). No hemoptysis, dyspnea, or desaturation were documented in our study.

\section{Discussion}

In fact, TACE has been widely performed for patients with unresectable HCC as it was reported by Kimura et al. [11]. For HCC management via TACE approach, knowledge of the hepatic vascular supply including extrahepatic collaterals is crucial not only for understanding the strategies' limitations but also to achieve optimal therapeutic response [12].

Sainani et al. [13] showed that hepatic tumor characterization on CT examination could be done carefully by the use of anatomical landmarks with contrast administration. Nowadays, awareness of the development of extrahepatic vascular collaterals by HCCs according either to tumor size and location or previous therapy such as surgical hepatic artery ligation or repeated chemoembolization is a prerequisite step [14].

Among these extrahepatic collaterals, the RIPA is the most common ectopic blood feeder of HCC, particularly those located at the hepatic dome [15, 22]. CT angiography with multiplanar reconstructions has become a potential non-invasive imaging technique for a variety of vascular districts. With this technique, a comprehensive quick evaluation of the RIPA anatomy can be accomplished and it can be useful in embolization planning [16].

In our study, all 58 cases were presented with hepatic dome HCC at the initial triphasic liver CT examination. According to the tumor size, the lesions were divided into three groups; $<5 \mathrm{~cm}, 5-10 \mathrm{~cm}$, and $\geq 10 \mathrm{~cm}$ in average diameters with the corresponding cases for each group; 12 (20.7\%), 39 (67.2\%), and 7 (12.1\%) respectively. The hepatic lesions of our cases were also divided into two groups regarding the tumor growth pattern; 23 cases (39.7\%) with and 35 cases $(60.3 \%)$ without liver capsule involved. The principle of involving liver capsule referred to the exophytic lesion beyond the hepatic contour. Our findings were matched with that were drawn by Sneag et al. [17].

On CT angiography, we defined ectopic tumor feeders within our cases as the vascular structures that were noticed adjacent to or penetrating the tumor. While Okino et al. [18] reported that the extrahepatic RIPA supplying HCC was suggested when the distal portion of the RIPA was observed near the liver irrespective of the relationship of the artery and tumor.

The right inferior phrenic artery usually originates from the celiac axis or directly from the aorta as a common trunk with the left inferior phrenic artery or independent origins [19]. Within our studied cases, 30 out of 33 (90.9\%) with an ectopic RIP arterial supply to HCC, the RIPA has a direct origin from the abdominal aorta with only three cases $(9.1 \%)$ from the celiac trunk.

Established risk factors predisposing to extrahepatic collateral arteries (ExCAs) feeding HCC include large tumors $(>5 \mathrm{~cm})$, peripherally located tumors, or exophytic tumor growth should be kept under consideration when reviewing imaging or management planning [20]. 

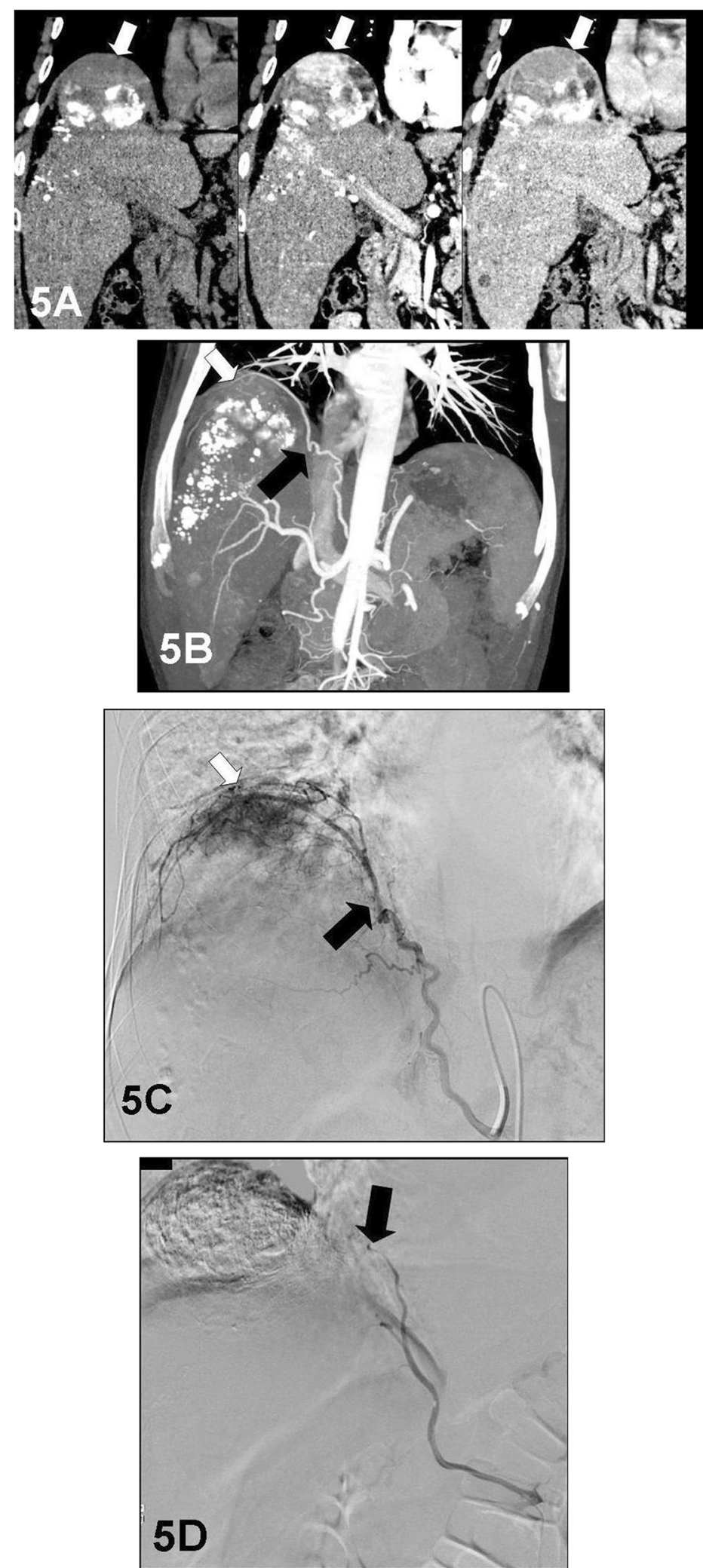

Fig. 5 (See legend on next page.) 
(See figure on previous page.)

Fig. 5 Follow-up Multi-phase CT liver after TACE showing a a managed hepatic dome HCC with an exophytic growth pattern displaying scattered lipiodol with a large residual/recurrent active lesion at its cephalic portion (arrows). b Coronal MIP showing the ectopic arterial supply of the active lesion by the right inferior phrenic artery (arrows). c Selective conventional angiogram of the right inferior phrenic artery (white arrow) of the same patient showing arterial blush (black arrow) of the active lesion at the cephalic portion of the managed hepatic dome HCC. $\mathbf{d}$ Postembolization proximal right phrenic angiogram with catheter tip at the vessel origin, demonstrating a lack of subdiaphragmatic

tumor hypervascularity

To Gokan et al. [21] and Zhao et al. [22], the main reason for RIPA supply to HCC is the anatomic tumor location adjacent to the ligaments suspending the liver at the bare area. The RIPA courses on the inferior surface of the diaphragm and consequently comes in a direct contact with the hepatic dome area. Our results appreciated this finding as within our 58 cases with hepatic dome HCC, 33 cases (56.9\%) were presented on CT angiography with extrahepatic collateral RIPA supply.

Our study revealed that the larger tumor size, the higher the prevalence of an extrahepatic arterial supply to HCC as 29 out of 33 cases (87.9\%) with an ectopic RIPA supply were presented with a tumor size in the range of $5-10 \mathrm{~cm}$, with its incidence reaching about $50 \%$ of our total 58 cases. No evidence of an ectopic blood supply to HCC smaller than $5 \mathrm{~cm}$ had been encountered within our cases. This was similar to the study performed by Chung et al. [12] as the probability of ExCAs in patients with a large tumor $(>5 \mathrm{~cm})$ was significantly higher than that for those patients with a small tumor $(<5 \mathrm{~cm})$.

Kim et al. [7] insisted that the exophytic tumor growth pattern was also a remarkable factor for the extrahepatic RIP arterial supply. As an exophytic HCC has a tendency to directly invade the diaphragm, so it can recruit a parasitic blood supply from the adjacent RIPA. In our study, 19 of 33 cases (57.6\%) with extrahepatic RIPA supply, tumor displayed an exophytic component with a relatively high incidence $(32.8 \%)$ within the total 58

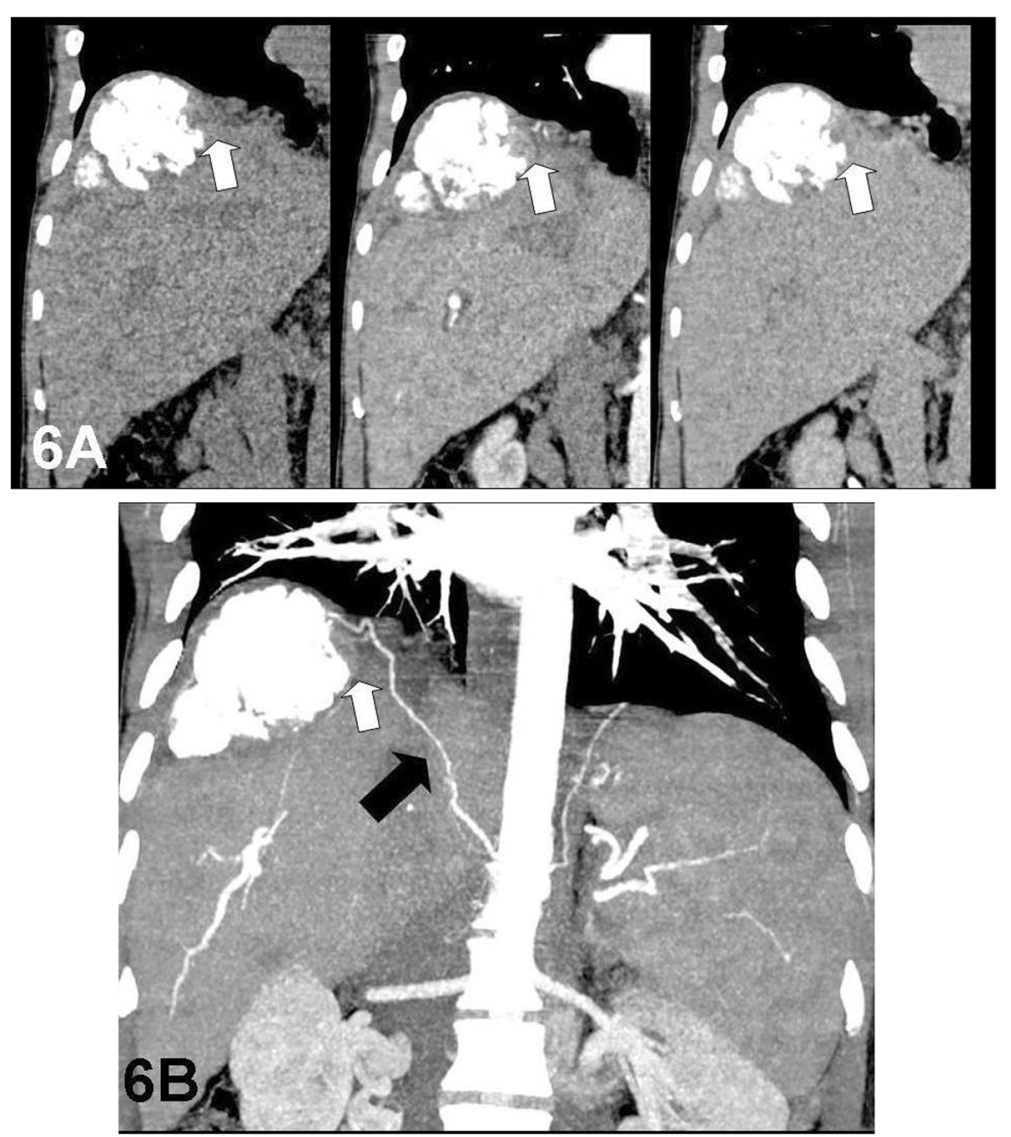

Fig. 6 Follow-up multi-phase CT liver after TACE showing a lipiodol retention with small residual/recurrent activity (white arrows) at the medial portion of the managed lesion at the hepatic dome. $\mathbf{b}$ Coronal MIP showing arterial supply of the active portion (white arrow) of the lesion by the right inferior phrenic artery (black arrow) 

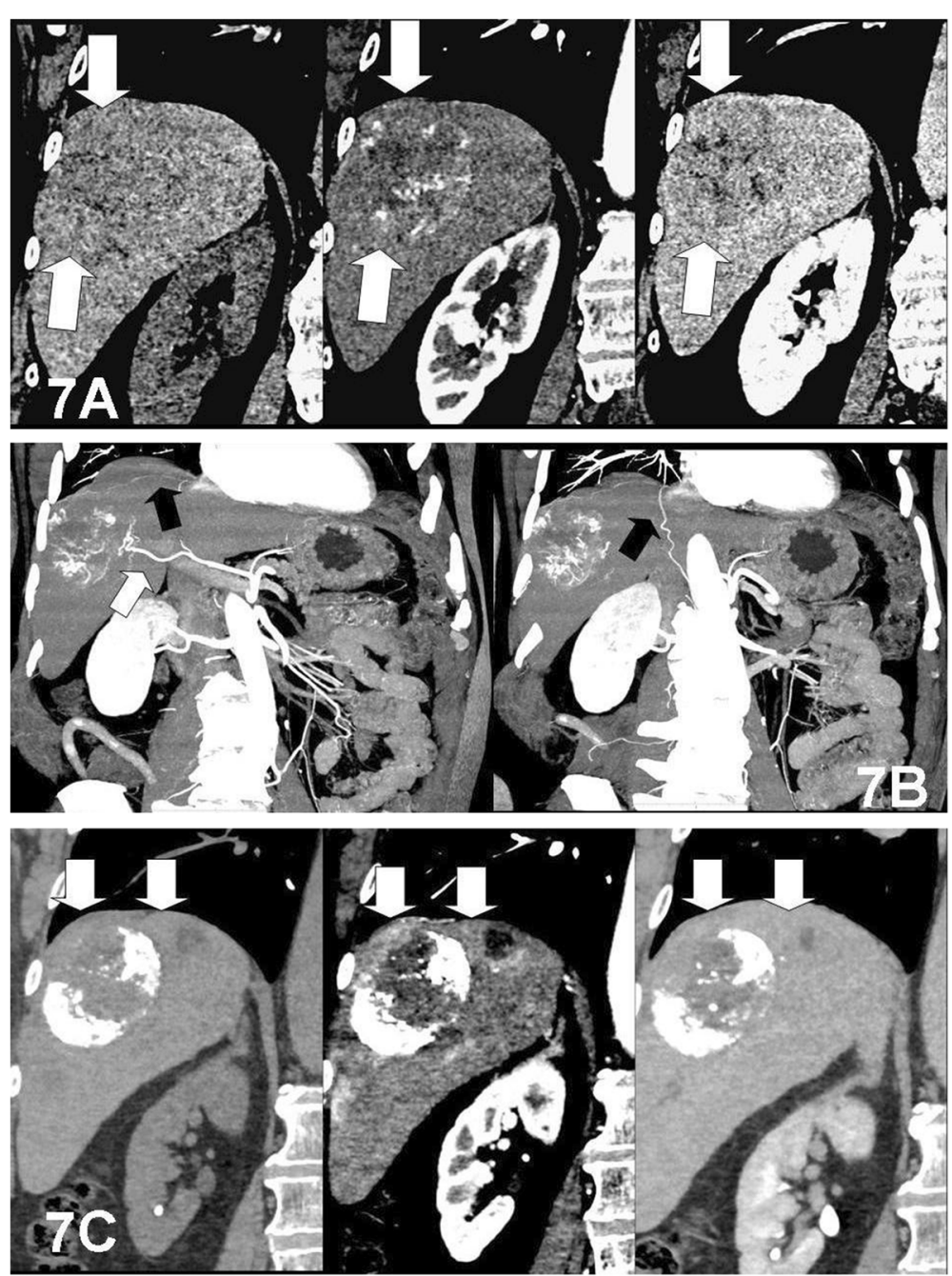

Fig. 7 Initial multi-phase CT liver with a coronal non-contrast, arterial and delayed phases showing a large hepatic dome HCC (white arrows) with heterogeneous arterial enhancement and delayed wash-out. b Coronal MIP showing arterial supply of the lesion by both the right hepatic artery (white arrow) and by extra-hepatic right inferior phrenic artery (black arrow). c Follow-up CT, 6 months after TACE without RIPA embolisation, showing residual activity at the cephalic portion of the lesion with progression of the lesion to the nearby liver parenchyma (white arrows) with lipiodol retention and inactive caudal portion

cases. Therefore, the exophytic growth pattern of $\mathrm{HCC}$ was considered predictive of ExCA formation.

According to the tumor therapy, all our cases with hepatic dome HCC were managed by TACE approach. Within the total cases, $26 / 58$ cases $(44.8 \%)$ only showed a history of repeated chemoembolization. We found that 22 out of 26 cases $(84.6 \%)$ with a history of repeated embolization sessions presented with extrahepatic collateral RIPA feeding HCC. This is in agreement with the studies carried out by Kim et al. [7] and kimura et al. [11] who reported that patients with repeated chemoembolization sessions usually presented by an extrahepatic collateral arterial supply to HCC even small-sized tumors as a result of attenuated lumen of the hepatic artery.
The study was performed by Sunwoo et al. [19] which widely appreciated that the extrahepatic vascular supply play a major role in tumor recurrence after receiving repeated chemoembolization. Once the number of TACE approaches has increased, the incidence of development of extrahepatic arteries has also increased as proved in our study.

In the present study, 25 out of 33 cases (75.8\%) with extrahepatic RIPA supplying hepatic dome HCC were undergone embolization of both hepatic artery and extrahepatic RIPA. Follow-up triphasic liver CT examination of the previous cases revealed good prognosis with lipiodol deposition along the tumor and no evidence of residual or recurrent active lesions 3-6 months following TACE procedure. While the remaining eight cases 

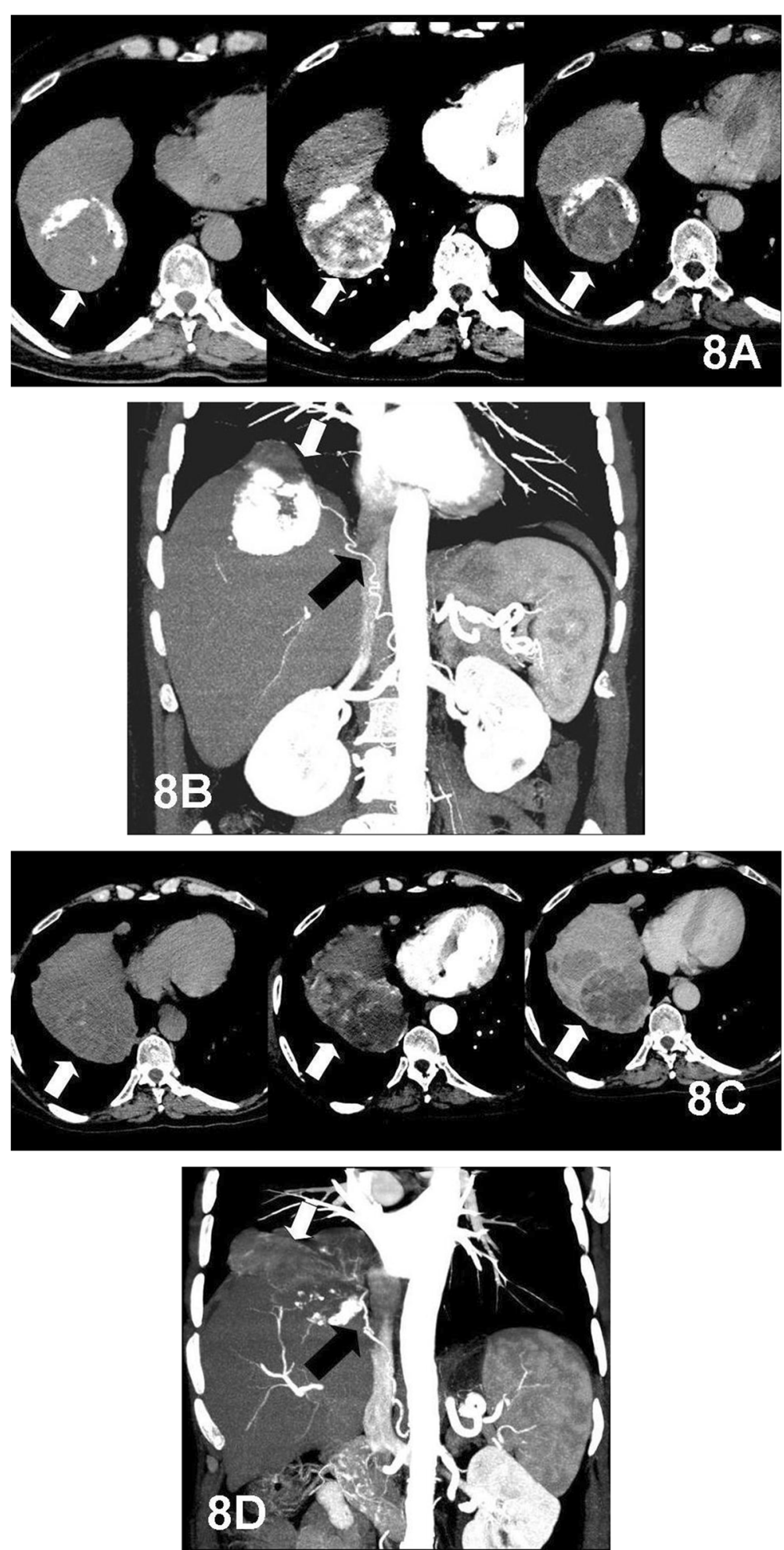

Fig. 8 Follow-up multi-phase CT liver after TACE showing a lipiodol retention with residual/recurrent activity (white arrows) of the managed lesion at the hepatic dome with partial exophytic component. b Coronal MIP showing the arterial supply of the active portion (white arrow) obtained by the right inferior phrenic artery (black arrow). c Follow-up CT, 3 months later showing progression of the active portion (white arrows), due to failure to identify the extra-hepatic collateral arterial supply before TACE. $\mathbf{d}$ Coronal MIP of the same study confirms the extrahepatic arterial supply of the progressive active portion of the lesion (white arrow) by RIPA (black arrow) 
(24.2\%) with only embolized hepatic artery (HA) and non-embolized RIPA showed progressive course of the active tumor residue that supplied by the extrahepatic RIPA. So, once this extrahepatic vascular feeder was identified, the patient should underwent TACE approach via the nutrient arteries to the tumor. Our findings were consistent with the study performed by Gwon et al. [16] that observed recognition of ExCAs is crucial for effective TACE procedure.

Our 25/33 cases with embolized RIPA during TACE procedure complained of right shoulder pain with about $23 / 25$ cases $(92 \%)$ complained of hiccups. All improved gradually within a few days with conservative treatment. Minimal right pleural effusion was observed in about 17 of 25 treated cases (68\%). No hemoptysis, dyspnea, or desaturation occurred. Watanabe et al. [23] showed nearly the same complications in HCC cases treated via embolization of the extrahepatic RIPA feeder. However, the previous study showed that one patient developed mild hemoptysis 4 days after TACE via the right IPA.

On the basis of our experience, the incidence of extrahepatic collateral arteries feeding $\mathrm{HCC}$ was positively associated with tumor size, peripheral tumor location, and the number of TACE sessions. In addition to the major vascular supply by hepatic artery, the most common EHA was the right inferior phrenic artery. This extrahepatic arterial feeder should be sought and identified in order to improve the effectiveness of TACE procedure.

We hope that this study has provided valuable information to interventionists, clinicians, and researchers alike by enhancing the understanding the anatomy of the RIPA and its potential significance in supplying hepatic dome HCC. In these cases, pre-interventional CT angiography with multiplanar reconstructions is fundamental in planning RIPA cannulation with a favorable prognosis.

\section{Conclusion}

TACE is an accepted therapy for the intermediate-stage HCC patients who are no longer candidates for surgical resection. Our study showed that EHC supply to HCC is not a rare finding particularly in patients with predisposing risk factors as repeated TACE sessions and when the HCC is large or peripherally located. Both diagnostic and interventional radiologists alike must be aware of this phenomenon as early detection represents the prerequisite for TACE success. So, when HCC is encountered in the hepatic dome area, a careful search for a tumor feeder by the RIPA on CT angiography should be performed before chemoembolization.

\section{Abbreviations}

MDCT: Multidetector computed tomography; CT: Computed tomography; TACE: Transarterial chemoembolization; RIPA: Right inferior phrenic artery; RIP: Right inferior phrenic; EHC: Extrahepatic collateral; ExCAs: Extrahepatic collateral arteries; IVC: Inferior vena cava; HCC: Hepatocellular carcinoma; AFP: Serum a-fetoprotein; CTA: CT angiography; MIP: Maximum intensity projection; MPR: Multiplanar reformation; EHAs: Extrahepatic arteries; HA: Hepatic artery; SPSS: Statistical Package for Social Science

\section{Acknowledgments \\ Not applicable.}

\section{Authors' contributions}

MSA carried out cases on workstation and selection of research cases, prepare the figures for cases demonstration, writing and review of the research. RAA: writing the research, sharing in selection of the cases; sharing in figures preparation and follow-up of cases. All authors read and approved the final manuscript.

\section{Funding}

This study had no funding from any resource.

\section{Availability of data and materials}

The datasets used and/or analyzed during the current study are available from the corresponding author on reasonable request.

\section{Ethics approval and consent to participate}

All procedures followed were in accordance with the ethical standards of the responsible committee on human experimentation "Institutional Review Board (IRB)" of National Liver Institute Menoufia University and with the Helsinki Declaration of 1964 and later versions. Committee's reference number is unavailable (NOT applicable). No consent was obtained from the patients since it was a retrospective study.

\section{Consent for publication}

All patients included in this research gave written informed consent to publish the data contained within this study.

\section{Competing interests}

The authors declare that they have no competing interests.

Received: 17 January 2020 Accepted: 17 March 2020

Published online: 23 March 2020

\section{References}

1. Kambadakone A, Baliyan V, Kordbacheh H, Uppot R, Thabet A, Gervais D, Arellano R (2017) Imaging guided percutaneous interventions in hepatic dome lesions: Tips and tricks. World J Hepatol 9(19):840-849

2. Chen G, Song B, Li Z, Yuan Y (2013) Ectopic blood supply of hepatocellular carcinoma as depicted by angiography with computed tomography: associations with morphological features and therapeutic history. PLoS ONE 8(8):e71942

3. Cazejust J, Bessoud B, Colignon N, Garcia-Alba C, Planché O, Menu Y (2014) Hepatocellular carcinoma vascularization from the most common to the lesser known arteries. Diagnostic and interventional imaging 95:27-36

4. Hiwatashi A, Yoshidaa K (2003) The origin of right inferior phrenic artery on multidetector row helical CT. Journal of Clinical Imaging:298-303

5. Wang Y, Li M, Cheng Y (2005) Influential factors and formation of extrahepatic collateral artery in unresectable hepatocellular carcinoma. World Journal of Gastroenterology 11(17):2637-2642

6. Moustafa S, Abdel Aal A, Ertel N, Saad N, DuBay D, Saddekni S (2017) Chemoembolization of hepatocellular carcinoma with extrahepatic collateral blood supply: anatomic and technical considerations. RadioGraphics 37:963-977

7. Kim H, Chung J, An S, Son K, Jae H, Park J (2008) Hepatocellular carcinoma: detection of blood supply from the right inferior phrenic artery by the use of multi-detector row CT. Vasc Interv Radiol 19:1551-1557

8. Özbülbül N, Yurdakul M, Tola M (2011) Does the right inferior phrenic artery have a supplying role in liver cirrhosis without hepatocellular carcinoma? A 64-slice CT study. Diagn Interv Radiol 17:239-242

9. Aslaner R, Pekcevik Y, Sahin H, Toka O (2017) Variations in the origin of inferior phrenic arteries and their relationship to celiac axis variations on CT angiography. Korean J Radiol 18(2):336-344

10. Basile A, Tsetis D, Montineri A, Puleo S, Saluzzo C, Runza G, Coppolino F, Ettorre G, Patti M (2008) MDCT anatomic assessment of right inferior 
phrenic artery origin related to potential supply to hepatocellular carcinoma and its embolization. Cardiovasc Intervent Radiol 31:349-358

11. Kimura S, Okazaki M, Higashihara H, Nozaki Y, Haruno M, H. Urakawa H, Koura S, Shinagawa Y, Nonokuma M (2009): Analysis of the origin of the right inferior phrenic artery in 178 patients with hepatocellular carcinoma treated by chemoembolization via the right inferior phrenic artery. Acta Radiologica, 48:7, 728-733.

12. Chung J, Kim H, Yoon J, Lee H, Jae H, Lee W, Park J (2006) Transcatheter arterial chemoembolization of hepatocellular carcinoma: Prevalence and causative factors of extrahepatic collateral arteries in 479 patients. Korean J Radiol 7(4)

13. Sainani N, Schlett C, Hahn P, Gervais D, Mueller P, Arellano R (2014) Computed tomography-guided percutaneous biopsy of isoattenuating focal liver lesions. Abdom Imaging 39:633-644

14. Ertreo M, Swerdlow D, Kim A, Somwaru A (2019) Extrahepatic Vascular Parasitization by a Hepatocellular Carcinoma. Cureus 11(9):e5807

15. Miyayama S, Matsui O, Taki K (2006) Extrahepatic blood supply to hepatocellular carcinoma: Angiographic demonstration and transcatheter arterial chemoembolization. Cardiovasc Intervent Radiol 29:39-48

16. Gwon D, Ko GY, Yoon H (2007) Inferior phrenic artery: Anatomy, variations, pathologic conditions, and interventional management. Radiographics 27:687-705

17. Sneag D, Krajewski K, Giardino A, O'Regan K, Shinagare A (2011) Extrahepatic spread of hepatocellular carcinoma: spectrum of imaging findings. AJR Am J Roentgenol 197:658-664

18. Okino Y, Kiyosue H, Matsumoto S, Takaji R, Yamada Y, Mori H (2003) Hepatocellular carcinoma: prediction of blood supply from right inferior phrenic artery by multiphasic CT. J Comput Assist Tomogr 27:341-346

19. Sunwoo L, Kim H, Yoo R, Chung J (2014) Chemoembolization via the right inferior phrenic artery in a patient with celiac stenosis: usefulness of angiography at full inspiration. Gastrointest Interv 3:58-60

20. Kim H, Chung J, Choi S, Jae H, Lee W, Park J (2007) Internal mammary arteries supplying hepatocellular carcinoma: Vascular anatomy at digital subtraction angiography in 97 patients. Radiology 242:925-932

21. Gokan T, Hashimoto T, Matsui S, Kushihashi T, Nobusawa H, Munechika H (2001) Helical CT demonstration of dilated right inferior phrenic arteries as extrahepatic collateral arteries of hepatocellular carcinomas. J Comput Assist Tomogr 25:68-73

22. Zhao Y, Fang Z, Luo J, Liu Q, Xu G, Pan H, Wei W, Yan Z (2015) Evaluation of extrahepatic collateral arteries in hepatocellular carcinoma in three independent groups in a single center. Experimental and therapeutic medicine 10:2366-2374

23. Watanabe Y, Tokue H, Taketomi-Takahashi A, Tsushima Y $(2018,5)$ Imaging findings and complications of transcatheter interventional treatments via the inferior phrenic arteries in patients with hepatocellular carcinoma. European Journal of Radiology Open:171-176

\section{Publisher's Note}

Springer Nature remains neutral with regard to jurisdictional claims in published maps and institutional affiliations.

\section{Submit your manuscript to a SpringerOpen ${ }^{\circ}$ journal and benefit from:}

- Convenient online submission

- Rigorous peer review

- Open access: articles freely available online

- High visibility within the field

- Retaining the copyright to your article

Submit your next manuscript at $\boldsymbol{\nabla}$ springeropen.com 